\title{
Editorials
}

\section{Embracing uncertainty to advance diagnosis in general practice}

In general practice, an accurate and rapid diagnosis could be obvious (herpes zoster), or essential (sepsis). More often, however, diagnosis in general practice is characterised by uncertainty. This may be because time is necessary for a particular condition to declare itself, or alternatively, that the problem will be self-limiting. The picture may be clouded by multimorbidity, as well as by culturallyshaped perceptions, interpretations, and presentations of symptoms.' We argue, however, that diagnostic uncertainty is not, as Jones has suggested, the new Achilles' heel of general practice, to avoid at all cost. ${ }^{2}$

We maintain instead that uncertainty typifies the nature and complexity of clinical knowledge, and is particularly salient in general practice. ${ }^{3}$ Diagnostic uncertainty deserves attention; not as evidence of sloppy practice, or professional failure, but as an inherent feature of, and condition for, advanced medical diagnosis. The nature of clinical knowledge rests on interpretation and judgment of bits and pieces of information which will always be partial and situated. ${ }^{4}$ In this commentary, we argue that the quality of diagnosis in general practice is compromised by believing that uncertainty can, and should, be eliminated.

On the contrary, we suggest, appropriate management of intrinsic uncertainty is a core clinical skill, which cannot be obtained from an essentialist attitude to knowledge where certainty is taken for granted as the standard. Only by embracing uncertainty as a predictable and inevitable companion of general practice, ${ }^{5}$ will the GP be able to meet the clinical challenges and develop the proficiency needed for diagnostic work in the primary care context. We endorse Jones' calls for improved diagnostic decisionmaking, ${ }^{2}$ but we advocate a closer look at the uncertainty that he seeks to eliminate. As experienced practitioners, we propose a fundamentally different foundation for improved decision making, with uncertainty as a vital and essential component of the diagnostic process. To make this case, we highlight some of the most obvious sources of uncertainty in general practice diagnosis.

\section{SOURCES OF UNCERTAINTY}

Diagnostic assessment takes place between individuals but is based on general categories. The GP makes judgements about similarity and difference, and finally determines that the case more closely fits

\author{
"Diagnostic uncertainty deserves attention; not as \\ evidence of sloppy practice, or professional failure, \\ but as an inherent feature of, and condition for, \\ advanced medical diagnosis.
}

the case description of one diagnosis than of another. This generalisation, is a pursuit of similarity and a cornerstone for clinical practice. Without it, we would have to treat each case empirically, without the benefit of the generalisation offered by classification. But, on the other hand, the practitioner encounters the challenge that Clinical medicine itself has to apply these laws to a particular patient with a unique history.'

The uniqueness of illness experience may be neglected by medicine in the pursuit of diagnostic certainty, while the patient's grayscaled narrative is transformed into a blackand-white diagnosis. ${ }^{5}$ The complexity of the particular fuels diagnostic uncertainty.

\section{TALKING ABOUT DISEASE}

The patient's story is essential for diagnostic work, with his or her perception and presentation of symptoms as the point of departure. ${ }^{1}$ The social implications of symptoms affect the presentation. Patients may have reasons to attend to some symptoms and keep others to themselves, as symptoms affect the way patients see themselves and the way they are perceived by others. They are, for example, more apt to present physical symptoms to their GP than those related to mental health.' Similarly, the GP's sympathy and empathy towards the patient, including previous experiences and stereotypes, will also affect their diagnostic perception and interpretation. This subjectivity and cultural frameworks are essential aspects of clinical interaction. Diagnostic judgement rests on interpretation in the cultural context, including issues of legitimacy regarding health, in the healthcare system and among lay people.
Communication and culture may also contribute to diagnostic uncertainty.

But uncertainty is not limited simply to the patient's story. The underlying models of disease are social and dynamic entities. ${ }^{8}$ Patients presenting symptoms that do not fit the patterns of biomedical diagnoses may challenge the GP. Women, as one example, often present completely different and lesscharacteristic symptoms of cardiovascular disease than men. Similarly, patients with medically unexplained symptoms, suffering from subjective symptoms without objective findings, also contest the disease model. And, of course, diagnostic concepts are in constant flux, undergoing change based on the use of new technology and epidemiology, creating new ways of descriptions and classification of bodily phenomena attached to new ways of treatment. Such mechanisms further contribute to the uncertainty of diagnosis.

\section{THE COMPLEXITIES OF GENERAL PRACTICE DIAGNOSIS}

A sophisticated understanding of probability is fundamental to assess the likelihood of a specific diagnosis, including the issue of urgency. Diagnostic tests themselves are fallible, and have varying degrees of reliability. Furthermore, interpretation of test results must take the epidemiological context into consideration due to the association between disease prevalence and the predictive values of tests. The positive predictive value of a pathological liver test result is for example, stronger in a gastroenterological department of a hospital compared to the predictive value of the identical numerical result in general practice, where liver diseases occur less

\section{'The complexity of the particular fuels diagnostic uncertainty.}




\section{"... it is time to develop theoretical, clinical and practical strategies for embracing - not simply tolerating - uncertainty.}

frequently. Hence, extensive testing does not eliminate uncertainty, rather the opposite as it introduces false positive and negative results.

In his Skinner lecture in 1942, Cohen discussed the nature, methods, and purpose of diagnosis, arguing that All diagnoses are provisional formulae designed for action.?

So far we have shown that diagnosis in general practice is dynamic and complex, far from the linear, predictable process manageable by algorithmic thinking. Furthermore, the diagnostic conclusion is a social construction, not an accurate and inevitable fact. $^{8}$ In 1984, McWhinney discussed Kuhn's ideas about scientific understanding as a process taking big leaps when implicit consensus about basic preconditions for knowledge - paradigms demonstrate their deficiency. ${ }^{10}$ Phenomena indicating such deficiency were called anomalies by Kuhn. Presenting tangible and convincing anomalies from general practice, McWhinney argued that they were signs of insufficiency or breakdown of 'normal', taken-for-granted medical systems of knowledge or paradigms.

\section{ACQUIRING THE SKILLS TO APPRECIATE UNCERTAINTY}

We do not dismiss the significance and consequences of diagnostic errors. GPs can certainly improve their diagnostic skills in many ways, and improvement of decision support tools and skills may offer some support. The rationalist tradition, however, seeks to provide a world of apparent security where certainty is readily achievable. ${ }^{5}$ The substantial examples of uncertainty in medical diagnosis that we have presented, call for a more fundamental paradigm shift in how we view medical knowledge. Postnormal science is a philosophy emphasising the relationship between uncertainties and the impact of decisions in a system, in this case concerning medical diagnosis. ${ }^{11}$ These theories reveal the challenges arising when we realise that the traditional domain of science covers only a confined proportion of clinical knowledge, where uncertainty as well as the impact of decisions are high.

Clinical practice must therefore develop and rely on epistemological rules beyond prediction and accuracy, acknowledging uncertainty as an important feature of knowledge and decision making. Nowotny suggests the notion 'cunning of uncertainty' as a strategy where we get to know uncertainty and acquire the skills to live with it. ${ }^{12}$ Simpkin and Schwartzstein advocate tolerance of uncertainty. ${ }^{5}$ However, for a paradigm shift regarding diagnosis in general practice, we suggest it is time to develop theoretical, clinical, and practical strategies for embracing ${ }^{13}$ - not simply tolerating uncertainty, instead of unsuccessfully trying to eradicate or suppress it.

\section{Kirsti Malterud,}

Research Professor of General Practice, The Research Unit for General Practice and Section of General Practice, Department of Public Health, University of Copenhagen, Copenhagen, Denmark Research Unit for General Practice, Uni Research Health, Bergen, Norway; Department of Global Public Health and Primary Care, University of Bergen, Norway.

\section{Ann Dorrit Guassora,}

Associate Research Professor of General Practice, The Research, Unit for General Practice and Section of General Practice, Department of Public Health, University of Copenhagen, Copenhagen, Denmark.

\section{Susanne Reventlow,}

Professor of General Practice, The Research Unit for General Practice and Section of General Practice, Department of Public Health, University of Copenhagen, Copenhagen, Denmark.

\section{Annemarie Jutel,}

Professor of Health, Graduate School of Nursing, Midwifery and Health, Victoria University of Wellington, Wellington, New Zealand.

\section{Provenance}

Freely submitted; externally peer reviewed.

DOI: https://doi.org/10.3399/bjgp17X690941

\section{ADDRESS FOR CORRESPONDENCE}

\section{Kirsti Malterud}

Research Unit for General Practice, Kalfarveien 31, N-5018 Bergen, Norway.

E-mail: kirsti.malterudagmail.com

\section{REFERENCES}

1. Malterud K, Guassora AD, Graungaard $A H$, Reventlow S. Understanding medical symptoms: a conceptual review and analysis. Theor Med Bioeth 2015; 36(6): 411-424.

2. Jones R. After Achilles. Br J Gen Pract 2016; DOI: https://doi.org/10.3399/bjgp16X683869.

3. Malterud K. The art and science of clinical knowledge: evidence beyond measures and numbers. Lancet 2001; 358(9279): 397-400.

4. Haraway D. Situated knowledges; the science question in feminism and the privilege of partial perspective. In: Haraway D, ed. Simians, cyborgs, and women. The reinvention of nature. New York, NY: Routledge 1991 183-201.

5. Simpkin AL, Schwartzstein RM. Tolerating uncertainty - The next medical revolution? N Engl J Med 2016; 375(18): 1713-1715.

6. McWhinney IR. 'An acquaintance with particulars .... Fam Med 1989; 21(4): 296-298.

7. Kroenke K. Patients presenting with somatic complaints: epidemiology, psychiatric comorbidity and management. Int J Methods Psychiatr Res 2003;12(1): 34-43.

8. Jutel AG, Dew K. Social issues in diagnosis: An introduction for students and clinicians. Baltimore, MA: Johns Hopkins University Press, 2014.

9. Cohen $\mathrm{H}$. The nature, methods and purpose of diagnosis. Lancet 1943; 24(6227): 23-25.

10. McWhinney IR. Changing models: the impact of Kuhn's theory on medicine. Fam Pract 1984; 1(1): 3-8.

11. Ravetz J. The post-normal sciences of precaution. Water Sci Technol 2005; 52(6): 11-17.

12. Nowotny $\mathrm{H}$. The cunning of uncertainty Cambridge: Polity Press, 2016.

13. Engebretsen E, Heggen K, Wieringa S, Greenhalgh T. Uncertainty and objectivity in clinical decision making: a clinical case in emergency medicine. Med Health Care Philos 2016; 19(4): 595-603. 